\title{
Research on Design of Radar Online Automatic Test System
}

\author{
Li Wanling ${ }^{1,}$, Yang Sen ${ }^{2}$, Chen Peng ${ }^{2}$ and Zhang Lianwu ${ }^{1}$ \\ ${ }^{1}$ Ordnance Technology research institute. Shijiazhuang Hebei, China \\ ${ }^{2}$ Department of Missile EngineeringOrdnance Engineering CollegeShijiazhuang, China \\ azyh8282180@sina.com
}

Keywords: On-line Automatic Test System, Signal Insulation, ATML Standard, Virtual Instrument

\begin{abstract}
Considering high false alarm probability of BIT and oversize of off-line ATE, the paper studies and designs an online automatic test system. The interface signals of radar line replaceable units (LRU) on working condition are leaded out by three-port conversion card and conditioned with insulation technology, making the effect of the test system on radar minimum. Then the signal is acquired by virtual instruments based on PXI bus. On software, the description document structure based on ATML standard is designed to describe test information and parsed to binary tree for checking and revising with recursive algorithm. At last, the data acquisition is achieved by LabWindows/CVI programming, calling description documents. Experiments verify the well test performance and efficiency of the test system.
\end{abstract}

\section{Introduction}

The equipment testability mainly include two meanings on built-in test (BIT) and automatic test equipment (ATE), As technology developed, BIT and ATE integrate day by day[1]. On the one hand, ATE moving towards universal, modularization, integration, miniaturization and even system on chip. On the other hand, BIT is more powerful, having a lot of function which only ATE equipment owned, such as fault detection, isolation and positioning etc[2]. But in the current, whether BIT or ATE both have deficiencies, BIT cannot accomplish all testing and diagnosis function, ATE also have not reached the level of integration and BIT miniaturization[3,4]. The practical application of BIT and ATE combination test is discussed by Literature [4], But in the process of combination, offline way is used by the ATE test, and a lot of drive source need to be provided, So the existence of hardware further integration of leeway. For this reason, in this paper, the online test system is designed in order to test and diagnosis equipment, that making up for the lack of offline ATE.

\section{The Overall Design and Demand Analysis}

Test System Structure. Nowadays more modular design is used by the current new radar.The whole diagnostic test system is shown in Figure 1. This paper studies online ATE hardware and software design, collecting the interface leads to a general signal and BIT signals with the acquisition, saving in accordance with the different levels, and finally providing fusion system to fault diagnosis of the test results.

\section{Test Requirement Analysis}

A radar transmitter and receiver of the letter at the other systems are use of standardized, modular design, the connection between the plug LRU and radar interface design using a common 200-pin connector or 26-pin air outlet. From the plug-in pin leads to signal conditioning circuit modules and LRU connection can use a common interface, which makes the test system hardware adapter circuit versatility greatly enhanced.

plug-in on the radar, according to their function, different pin signal can be divided into several categories. Including the DC signal and AC signal, 12bit parallel bus signal, clock signal square wave and sine signal, the timing pulse signal, the serial bus signals, the switch level, sinusoidal modulation of the quadrature signal, etc. Many signals have triggered synchronization or mutual relations, in the 
test design must include the testing of various types of signal hardware and software design, test access and coordinate different types of signals, synchronization signals and trigger. Design software, taking into account the complex signal types, the large number of signals also need to configure the instrument configuration and results are saved for special management.

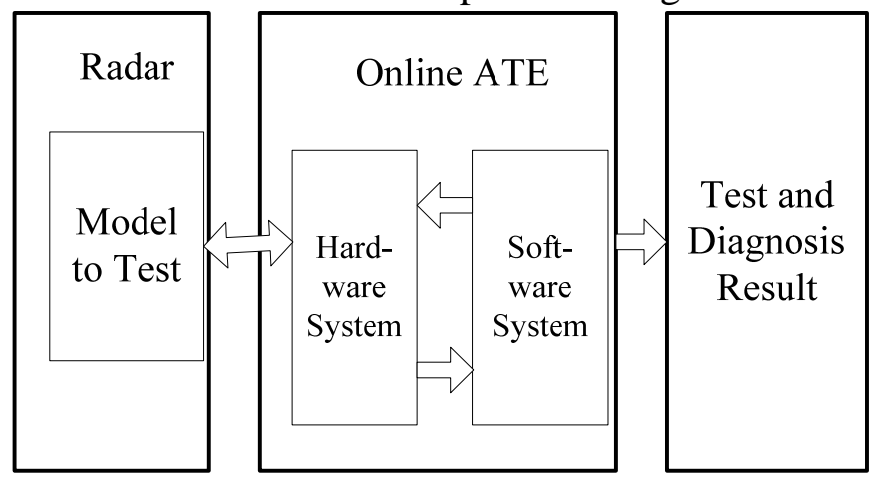

Fig.1 Architecture of Radar Online Test System

\section{Hardware System Design}

The hardware acquisition system is divided into four parts: Universal three-way adapter plate, special conditioning board, check the switch board, PXI bus test resources. Here taking a typical signal process system as an example.

\section{Conditioning Board Design}

Universal Three-way Adapter Plate. This adapter board has two functional areas: 200 core connector adapter area and the air outlet adapter. It is Showed in Figure 2.

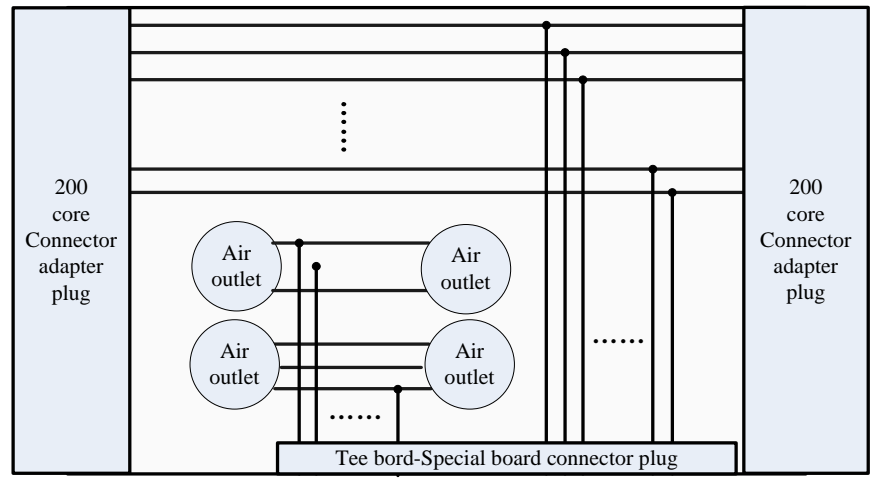

Fig.2 Architecture of the generic three-way board

This adapter plate mainly completes the three links, in order to lead the signal out. The adapter plate using the four-layer board design, top-level cloth quotes lead, the second layer laid to land. Without Power planes, the third and the fourth floors are laid LRU and radar backplane cable. The third or fourth layers of the adapter plate both are parallel lines, it is easy to form the crosstalk. Especially when the adjacent clock line or the reset line exist, and the crosstalk is serious may cause the radar LRU trouble-free does not work properly. Taking into account of the trace impedance and the inherent inductance of the equipment, the Routing need to be bold designed.

Special Conditioning Board Design. (1) Streamlining and consolidation of the interface signals.Before designing the Special conditioning board, the LRU interface signals need to be concluded and optimized, the final comprehensive radar extension LRU module interface signals designed for multiple LRU dedicated conditioning board.

(2) Signal isolation. Signal isolation, isolation leads to three links board signal to treat the system of measuring equipment. For analog signal using the oscilloscope front-end amplifier design shown in Figure 3.The purpose of this circuit is to improve the input impedance, and reduces the input current to reduce the output impedance and improve the drive capability of the output stage. 


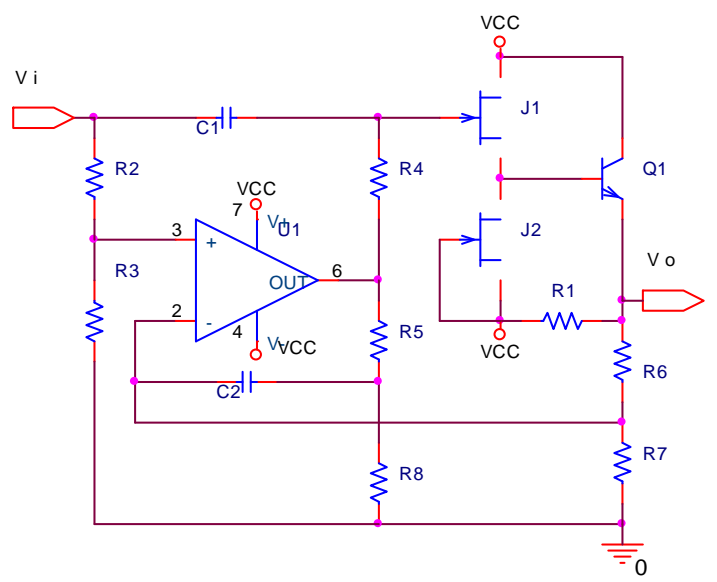

Fig.3 Design of Analog signal isolation

\section{Software System Design}

ATML Standard. ATML standard system provides for the one used for the integration of design data, test strategies and requirements, test procedures, test results and test systems to achieve integrated development environment. The standard system involves the IEEE 1232TM (AI-ESTATE) standard,the IEEE P1636TM (SIMICA), IEEE Std 1522TM (Testability and Diagnosability Characteristics and Metrics) and the IEEE Std 1641TM (STD) standard[5].

The Design of Software Architecture Based on ATML. The test software based on ATML is designed according to the needs of the actual system design and test, the structural model is showed in Figure 4.

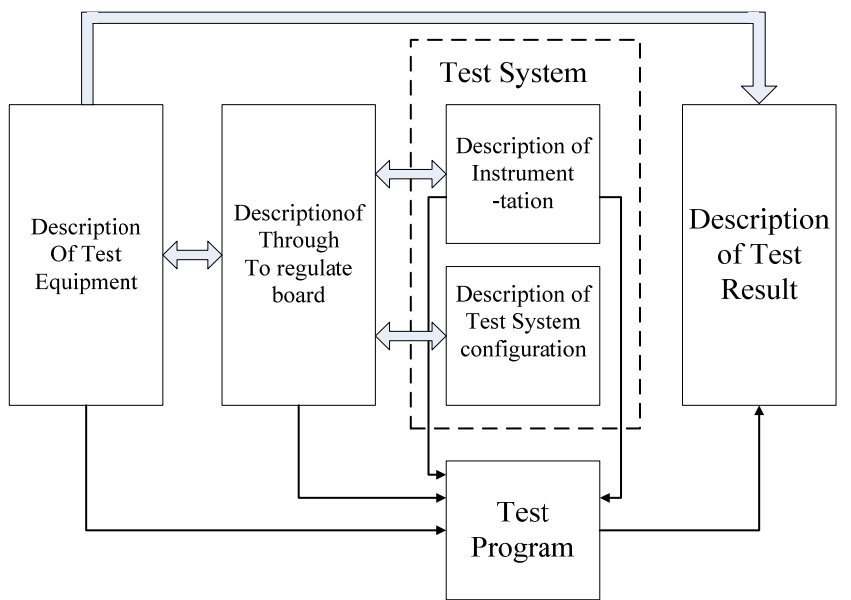

Fig.4 The Design of Software Architecture Based on ATML

This system description document models contain the test equipment description document, transfer conditioning board description document, instrument configuration description document, the test system configuration description document and the test results description document[6].

\section{Parsing of the ATML Description Document}

Description of the document are based on ATML or modify the schema design and fill out the test procedures in reference to the document needs to parse the document. The approach adopted here is to design a binary linked list data structure, after reading the XML documents ,we use a recursive algorithm to save the information in the data structure, then query the node in the list of node identification information.

Such as the hierarchy of an XML document in Figure 8 shows on the left, the document specified in the parsed into a binary tree node with the same level of information to the left child node, sub-level information to the right child node. And so on, the result of XML documents analytical shows on the right of Figure 5. 


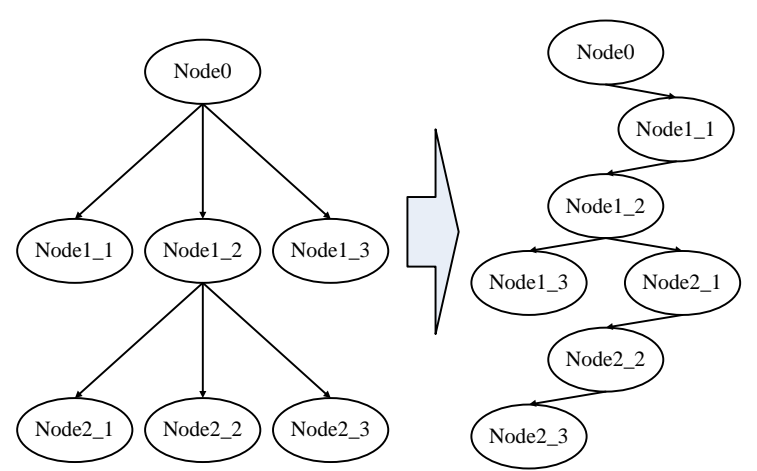

Fig.5 The Transform of XML Document Parsing Data

Recursively nested method is used when implementing the software, and if we can not analysis the child nodes from the parent nodes ,we need to jump from function to terminate the current function nesting level. LabWindows/CVI cvixml.dll function is called in the implementation process to achieve the operation of an XML document.

\section{Summary}

In this paper, we use the test system testing the key signal of a certain type of radar LRU interface, research and design of the online test system solving the offline ATE with too large volume, too complex structure problems, achieving a combination test of the BIT and online ATE. The test system achieves the online isolation of analog and digital signals by using the test signal isolation technology, and making the online test effects on the equipment work reduce to a minimum. Using ATML standardization description in the instrument software greatly increase the flexibility, portability and efficiency development of software programming, and it make test systems have a certain versatility and better maintainability, in practical work it achieves good results.

\section{References}

[1] Xu Yong-cheng, Wen Xi-sen, Yi Xiao-shan, Liu Guan-jun.The analysis of Built-in test technology development trend [J].Measurement\&Control technology ,2001, 20(8),1-4.

[2] Yang Xue-xian.Research and realize of Radar system Online detection technology [D].Beijing;Beijing Institute of Tecchnology, 1999,13

[3] Ma Ding,Wang Yuan-da,Lu Yong-ji.Seeing the development trend of ATE from the changing of air weapons and equipment maintenance system [J].Aeronautical science and technology. 2008,5,11-14

[4] Jiang Jing,Zhang Xiao-ping,Sun Xiao-shu.BIT and ATE combination test technology and its applications [J].2006,3(21),383-385

[5] IEEE. IEEE Std 1671. IEEE Trial-Use Standard for Automatic Test Markup Language (ATML) for Exchanging Automatic Test Equipment and Test Information via XML[S] . 2006.

[6] IEEE. IEEE Trial-Use Standard for Automatic Test Markup Language (ATML) for Exchanging Automatic Test Equipment and Test Information via XML: Exchanging Instrument Descriptions [S]. 2008 